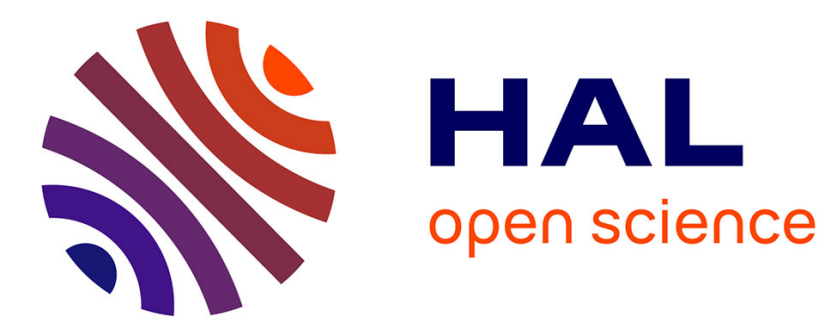

\title{
Comportement du béton sous fort confinement : effet du rapport eau/ciment
}

\author{
Xuan Hong Vu, Yann Malecot, Laurent Daudeville, Eric Buzaud
}

\section{To cite this version:}

Xuan Hong Vu, Yann Malecot, Laurent Daudeville, Eric Buzaud. Comportement du béton sous fort confinement : effet du rapport eau/ciment. European Journal of Environmental and Civil Engineering, 2008, 12 (4), pp.429-457. hal-02002529

\section{HAL Id: hal-02002529 \\ https://hal.univ-grenoble-alpes.fr/hal-02002529}

Submitted on 31 Jan 2019

HAL is a multi-disciplinary open access archive for the deposit and dissemination of scientific research documents, whether they are published or not. The documents may come from teaching and research institutions in France or abroad, or from public or private research centers.
L'archive ouverte pluridisciplinaire HAL, est destinée au dépôt et à la diffusion de documents scientifiques de niveau recherche, publiés ou non, émanant des établissements d'enseignement et de recherche français ou étrangers, des laboratoires publics ou privés. 


\title{
Comportement du béton sous fort confinement : effet du rapport eau/ciment
}

\author{
Xuan Hong Vu*, Yann Malecot*, Laurent Daudeville*, Eric \\ Buzaud** \\ * Laboratoire Sols, Solides, Structures - Risques \\ Université de Grenoble, B.P. 53 - 384041 Grenoble Cedex 9, France \\ vu@hmg.inpg.fr - yann.malecot@hmg.inpg.fr - laurent.daudeville@ujf-grenoble.fr \\ ** DGA, Centre d'Etudes de Gramat, 46500 Gramat, France
}

\begin{abstract}
RÉSUMÉ. Cette étude concerne l'identification du comportement du béton sous sollicitations triaxiales sévères (chutes de blocs, explosions, impacts balistiques). Pour reproduire de forts niveaux de contrainte avec des trajets de chargement bien contrôlés, des essais statiques sont réalisés sur des échantillons de béton en utilisant une presse triaxiale de très grande capacité (niveaux de contrainte de l'ordre du GPa). En outre, il est connu depuis longtemps que le rapport E/C, entrant dans la composition du béton, est un des paramètres majeurs conditionnant la porosité et la résistance de la matrice cimentaire du béton durci. L'objectif de cet article est donc de quantifier l'effet de ce rapport sur le comportement du béton sous fort confinement. A partir de la composition d'un béton de référence " ordinaire » $(E / C=0,6)$, deux autres bétons avec des rapports $E / C$ respectivement égaux à 0,4 et 0,8 sont formulés. Cet article présente les résultats des essais triaxiaux réalisés sur ces trois bétons. Ces résultats montrent que, contrairement à ce qui est observé en compression simple, le béton se comporte sous fort confinement comme un empilement granulaire sans aucune influence de la résistance de la matrice cimentaire.
\end{abstract}

ABSTRACT. This study concerns the identification of concrete behaviour under severe triaxial loadings (rock falls, explosions, ballistic impacts). In order to reproduce high stress levels with well controlled loading paths, static tests are carried out on concrete samples by means of a very high capacity triaxial press (stress levels of the order of the GigaPascal). In addition, it is known for a long time that the water/cement ratio (W/C), entering the concrete composition, is one of the major parameters conditioning the porosity and the strength of the cement matrix of the hardened concrete. The objective of this article is to quantify the effect of this ratio on the concrete behaviour under high confinement. From the composition of a reference "ordinary" concrete $(W / C=0,6)$, two other concretes were formulated with $W / C$ ratios equal to 0,4 and 0,8 respectively. This article presents experimental results concerning the effect of the water/cement ratio $(W / C)$ of the concrete on its behaviour under high confinement. The test results presented in this article show that under high confinement, the concrete behaves as a granular stacking which the concrete constitutes without any influence of the cement matrix strength.

MOTS-CLÉS : béton, rapport eau/ciment, essai triaxial, fort confinement.

KEYWORDS : concrete, water/cement ratio, triaxial test, high confinement. 


\section{Introduction}

Cette étude concerne l'identification du comportement du béton sous sollicitations extrêmes (explosions en champ proche ou impacts balistiques). Lors de telles sollicitations le béton est soumis à des états de contrainte triaxiaux d'intensité très importante. Par exemple, durant l'impact d'un projectile rigide sur une structure massive en béton, trois phases du comportement triaxial peuvent être observées. Chaque phase est associée à des modes d'endommagement différents mais se produisant parfois simultanément (Zukas, 1992), (Bailly et al., 1996). Sur la face avant de la structure, on constate une cratérisation associée d'un point de vue mécanique à de la traction simple. La pénétration du projectile au cœur de la structure génère ensuite une compression triaxiale, l'inertie de la structure créant un confinement passif dans la zone située autour du projectile. Enfin, si la cible est suffisamment mince, lors de la dernière phase de pénétration, on observe des contraintes de cisaillement et de traction simple sur la face arrière du béton. Dans les cas extrêmes l'impact peut se terminer par une perforation complète de la cible. Ainsi, la validation de modèles de comportement du béton prenant en compte simultanément les phénomènes d'endommagement fragile et de déformation irréversible comme la compaction nécessite de disposer de résultats d'essais reproduisant les chemins de sollicitation complexes décrits précédemment.

La plupart des résultats expérimentaux disponibles dans la littérature ne concernent que des chargements triaxiaux avec une pression de confinement modérée (Li et al., 1970) ; (Kupfer et al., 1973) ; (Wang et al., 1987) ; (Jiang et al., 1991) ; (Imran et al., 1996); (Taliercio et al., 1999) ; (Sfer et al., 2002). Ils ont notamment permis de mettre en évidence la transition de comportement fragileductile qui est une caractéristique des matériaux cohésifs frottants. Les études numériques montrent que les essais dynamiques sur béton, par exemple au moyen de barres de Hopkinson (1914) (Zhao et al., 1996) sont difficiles à réaliser essentiellement en raison du caractère fragile du matériau qui conduit à une rupture en phase transitoire. Le caractère peu homogène de l'état de contrainte dans l'échantillon, le contrôle très limité du trajet de chargement et une instrumentation relativement pauvre rendent l'exploitation des résultats d'essais le plus souvent délicate. Les résultats d'essais disponibles dans la littérature permettent cependant de mettre en évidence l'augmentation de la résistance du béton avec la vitesse de déformation. Cette dépendance du comportement du béton à la vitesse de déformation semble faible en l'absence de phase liquide (Bischoff et al., 1991); (Toutlemonde, 1995). Elle est en revanche très forte en traction et peut être en grande partie expliquée par l'influence des défauts (Hild et al., 2003). Le comportement triaxial dynamique du béton est un domaine très peu étudié. Des essais aux barres de Hopkinson ont été réalisés par Schmidt sur des échantillons de béton confinés jusqu'à $7 \mathrm{MPa}$. Ces essais montrent une diminution de l'effet de vitesse sur le comportement du béton lorsque le confinement augmente (Schmidt, 2003). Toujours en dynamique, un essai d'impact de plaques permet l'identification de l'équation d'état (pression - variation de volume) à très haut niveau de contrainte 
mais avec un coût d'expérimentation très élevé puisque chaque essai correspond à un point de la courbe de Hugoniot. Par ailleurs, ce type d'expérimentation génère des niveaux de contraintes généralement plus élevés que ceux obtenus avec des problèmes d'impacts classiques sur une structure massive en béton.

Les résultats présentés dans cet article concernent des essais triaxiaux statiques réalisés sur des échantillons de béton grâce à une presse triaxiale de grande capacité, dénommée presse « GIGA ». Ce dispositif expérimental permet d'atteindre, au sein des échantillons, des niveaux de contrainte moyenne de l'ordre du giga Pascal avec des trajets de chargements statiques, homogènes et bien contrôlés. Le fait de caractériser par des expérimentations quasi-statiques un modèle de comportement destiné à des calculs dynamiques n'est pas une pratique nouvelle dans l'étude des géomatériaux. Les études expérimentales similaires ont, jusqu'à ce jour, été limitées à des échantillons de mortier de faible dimension (Bazant et al., 1986); (Buzaud 1998); (Burlion et al., 2001); (Williams et al., 2005). Ils montrent notamment l'augmentation de la contrainte déviatoire maximale du mortier et l'évolution de son état limite avec le confinement. Il s'agit ici d'étendre cette pratique à l'étude de « vrais » bétons (taille de granulats d'ordre centimétrique). L'étude comparative entre un béton et un mortier à une pression de confinement de $500 \mathrm{MPa}$, effectuée par S. Akers (Akers et al., 2004), souligne les différences de comportement entre les deux matériaux, et montre que l'étude d'un mortier à fort confinement n'est pas représentative du comportement du béton. D'autres résultats d'essais triaxiaux sur béton ordinaire à des pressions de confinement comprises entre 0 et $500 \mathrm{MPa}$ montrent l'évolution du comportement et de l'état limite du béton avec le confinement (Schmidt, 2003); (Warren et al., 2004).

Depuis 2004, le laboratoire 3S-R s'est lancé en collaboration avec le Centre d'Etudes de Gramat (DGA) dans un vaste programme de recherche sur la vulnérabilité des infrastructures en béton. C'est dans ce cadre qu'un programme d'étude expérimentale sur un béton modèle a été défini. De manière concomitante aux travaux présentés ici et en utilisant le même matériau modèle, Gabet a étudié l'influence du trajet de chargement sur le comportement du béton (Gabet et al., 2007). Il a notamment montré que sous fort confinement l'état limite du béton est très peu dépendant à la fois du trajet de chargement et de l'angle de Lode.

Dans cet article, on s'intéresse plus spécifiquement à l'effet du rapport eau/ciment $(\mathrm{E} / \mathrm{C})$, entrant dans la composition du béton frais, sur le comportement $\mathrm{du}$ béton durci sous très fort confinement. L'effet du rapport $\mathrm{E} / \mathrm{C}$ sur la résistance $\mathrm{du}$ béton en compression simple est bien connu. Dans les règles de calcul des structures en béton, le béton est essentiellement caractérisé par sa résistance en compression simple à 28 jours $f_{c 28}$. La plupart des autres caractéristiques en sont déduites (résistance en traction, module de Young ...). Or pour un type de granulats et pour un type de ciment donnés, $f_{c 28}$ dépend presque exclusivement de la composition de la pâte de ciment c'est-à-dire du rapport E/C (Féret 1892), (Bolomey 1935), (Neville 1959), (Gilkey 1961), (Alexander et al., 1982), (Kakizaki 1992). Le rapport E/C conditionne aussi la porosité de la matrice cimentaire du béton durci (Roy et al., 
1973), (Sersale et al., 1991), (Chanvillard 1999) qui elle-même influe beaucoup sur la durabilité du béton (Ollivier et al., 1992). La dépendance du comportement triaxial du béton vis-à-vis du rapport $\mathrm{E} / \mathrm{C}$ est donc un facteur primordial à étudier. Des essais uniaxiaux confinés à forts niveaux de contrainte réalisés sur des mortiers pour différents rapports $\mathrm{E} / \mathrm{C}$ montrent que la compaction du matériau est fonction de sa porosité interne (Burlion 1997) ; (Burlion 2001). Ainsi, la compaction du mortier est d'autant plus importante que son rapport $\mathrm{E} / \mathrm{C}$ est grand. En outre, des essais triaxiaux à faibles niveaux de confinement (inférieurs à $15 \mathrm{MPa}$ ) mettent en évidence que la contrainte déviatoire, le module de Young et le module de compressibilité du mortier diminuent aussi lorsque son rapport $\mathrm{E} / \mathrm{C}$ augmente (Yurtdas et al., 2005). En revanche, à ce jour, il n'existe pas de résultat sur l'effet du rapport $\mathrm{E} / \mathrm{C}$ sur le comportement triaxial du béton sous fort confinement.

Le dispositif expérimental utilisé pour cette étude est décrit au paragraphe 2. Les résultats d'essais triaxiaux effectués sur trois bétons possédant des rapports $\mathrm{E} / \mathrm{C}$ différents sont ensuite présentés, d'abord en termes de courbes contraintedéformation (paragraphe 3) puis en termes de courbes d'état-limite (paragraphe 4). Ces résultats montrent que contrairement à ce qui est observé en compression simple et à faible pression de confinement, le comportement déviatoire du béton devient insensible au rapport $\mathrm{E} / \mathrm{C}$ pour de forts niveaux de confinement.

\section{Dispositif expérimental}

\subsection{Cellule triaxiale}

Une presse triaxiale de grande capacité a été spécialement conçue pour cette étude (Thiot 2004). La mise au point des essais sur béton qui s'est avérée délicate est présentée de manière détaillée dans le mémoire de thèse de $\mathrm{X}$. $\mathrm{H}$. Vu $(\mathrm{Vu}, 2007)$. La Figure 1 montre la vue générale de la presse GIGA. Une vue en coupe de la cellule de confinement est présentée sur la Figure 2. Cette presse est capable de solliciter des éprouvettes de béton cylindriques de $7 \mathrm{~cm}$ de diamètre et $14 \mathrm{~cm}$ de hauteur jusqu'à une pression de confinement de $0,85 \mathrm{GPa}$ et une contrainte axiale de 2,3 GPa (Figure 3). L'échantillon de béton est placé à l'intérieur de la cellule de confinement. Le fluide de confinement, le di-2-ethylhexyl azelate (DOZ), un composant organique non-volatile, inerte et peu compressible, est injecté dans la cellule à travers son bouchon supérieur. Il est ensuite mis en pression par l'intermédiaire d'un système de vérin multiplicateur. L'effort axial est généré par le biais d'un vérin d'une capacité de $13 \mathrm{MN}$ qui se situe sous la cellule. Il est transmis à l'échantillon par l'intermédiaire d'un piston qui passe à travers le bouchon inférieur de la cellule de confinement. Un capteur de déplacement positionné dans la presse est utilisé pour piloter le déplacement du vérin axial tandis qu'un capteur de force et un capteur de pression placés à l'intérieur de la cellule de confinement mesurent l'état de contrainte de l'échantillon. L'asservissement entre la pression de 
Béton sous fort confinement : effet du rapport eau/ciment 5

confinement et le déplacement du vérin axial permet de réaliser différents trajets de chargement.

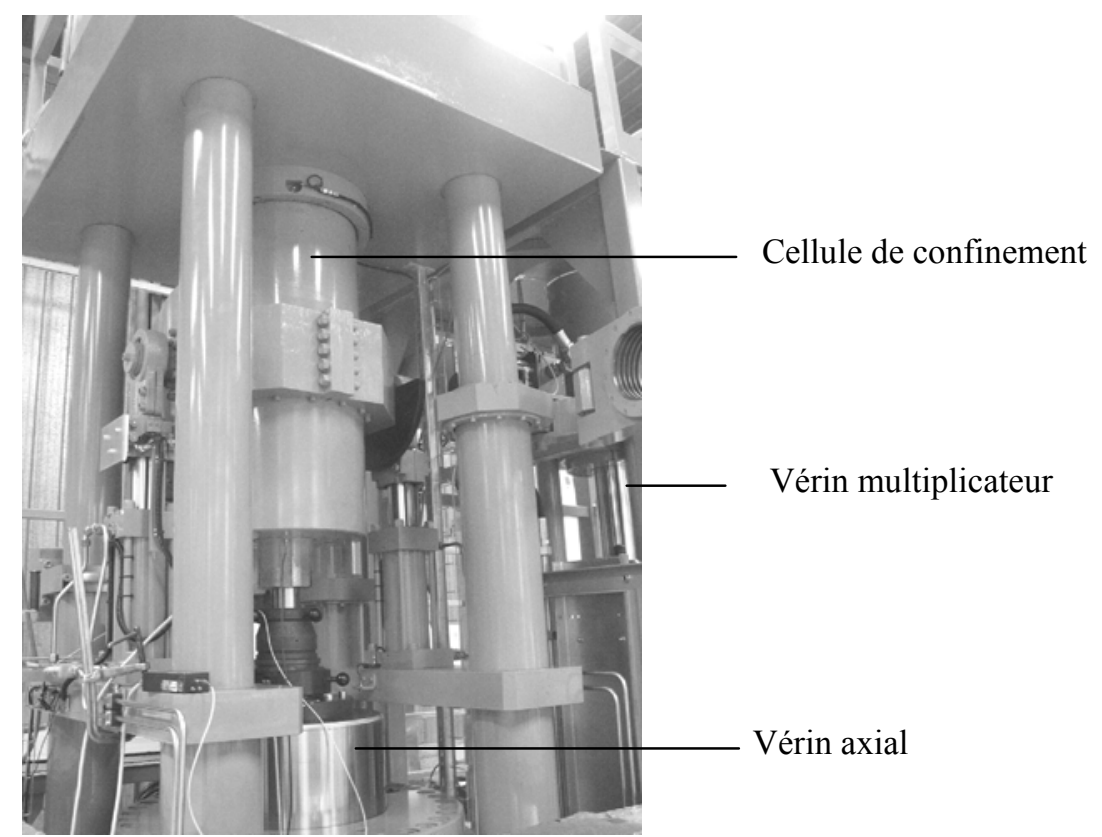

Figure 1. Vue générale de la presse GIGA 


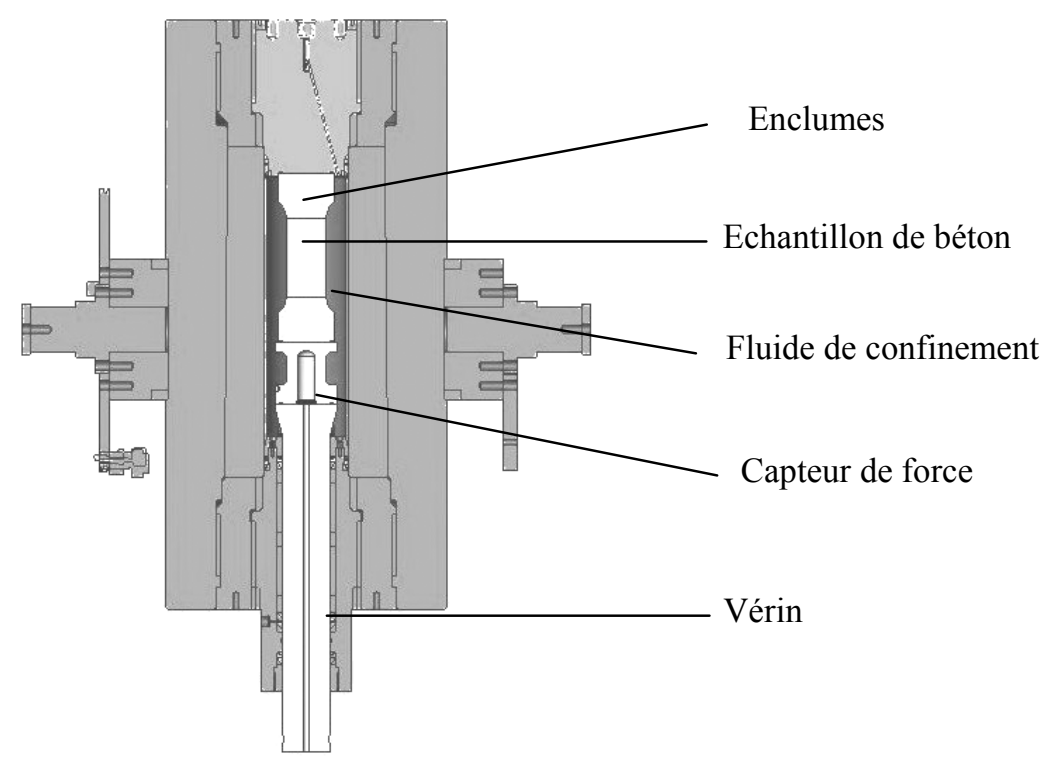

Figure 2. Vue en coupe de la cellule de confinement
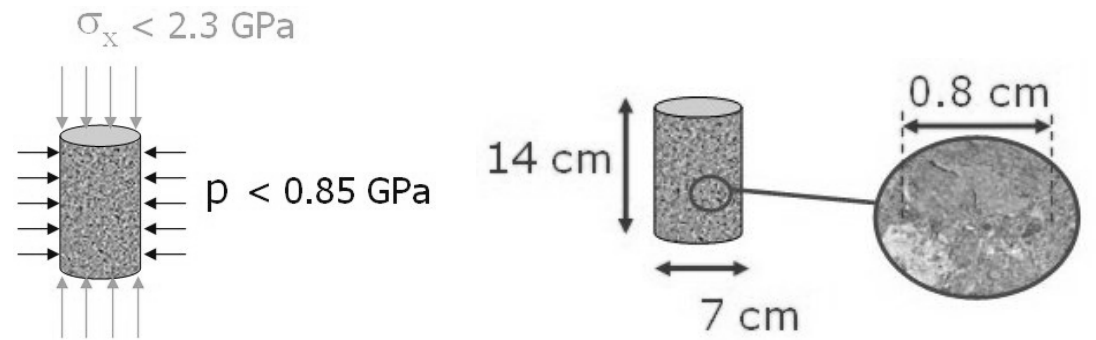

Figure 3. Capacité de sollicitation maximale de la cellule triaxiale et dimensions associées de l'échantillon

\subsection{Mesure des déformations}

La mesure des déformations est effectuée par l'intermédiaire d'un capteur LVDT axial, d'une jauge axiale et de deux jauges circonférentielles (Figure 4). Chaque extrémité du capteur LVDT est positionnée sur une enclume. Ce capteur permet donc d'obtenir la déformation moyenne de l'échantillon. La jauge axiale qui donne une mesure locale permet d'évaluer l'homogénéité de la déformation de l'échantillon. La déformation circonférentielle est mesurée à l'aide de deux jauges diamétralement opposées. Ces deux jauges augmentent la probabilité de garder au moins une mesure jusqu'à la fin de l'essai et permettent aussi de contrôler 
l'homogénéité de la déformation. L'utilisation de jauges pour des essais triaxiaux sur béton à des niveaux de confinement aussi importants est à notre connaissance originale.

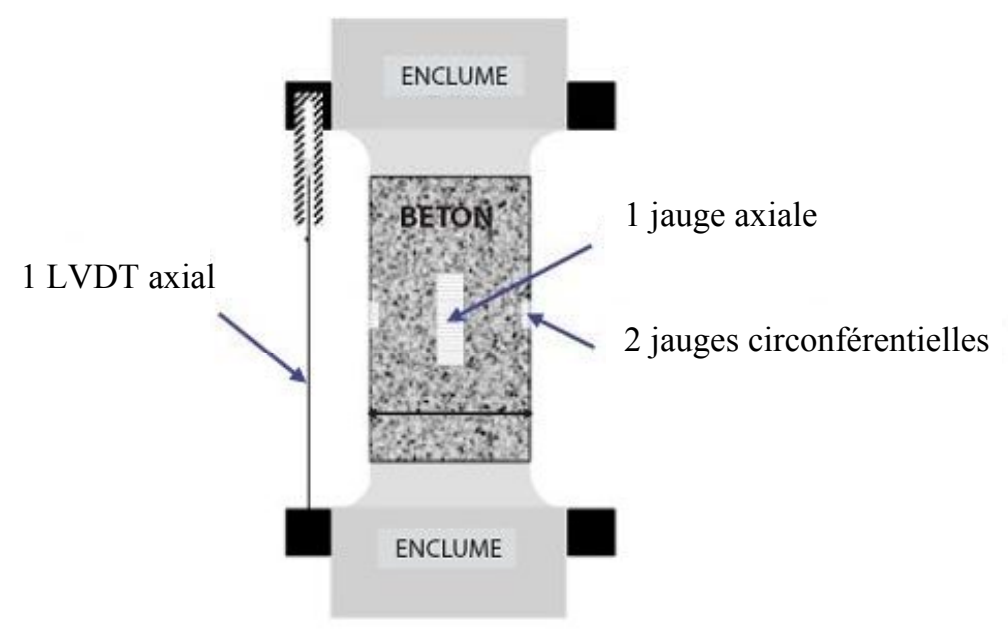

Figure 4. Schéma de principe de la mesure des déformations sur l'échantillon

\subsection{Protection de l’échantillon}

Compte tenu du caractère poreux du béton, le fort niveau de confinement a nécessité le développement d'une membrane de protection multicouche autour de l'échantillon. Celle-ci est constituée de $8 \mathrm{~mm}$ de latex et de $2 \mathrm{~mm}$ de néoprène (Figure 5). Elle empêche le fluide de confinement de pénétrer à l'intérieur de l'échantillon. Par ailleurs un bouclier plastique posé sur les jauges les protège contre un éventuel poinçonnement de la membrane (Vu et al., 2005). La figure 5 montre le bouclier ainsi que la membrane mis successivement en place autour de l'échantillon. 

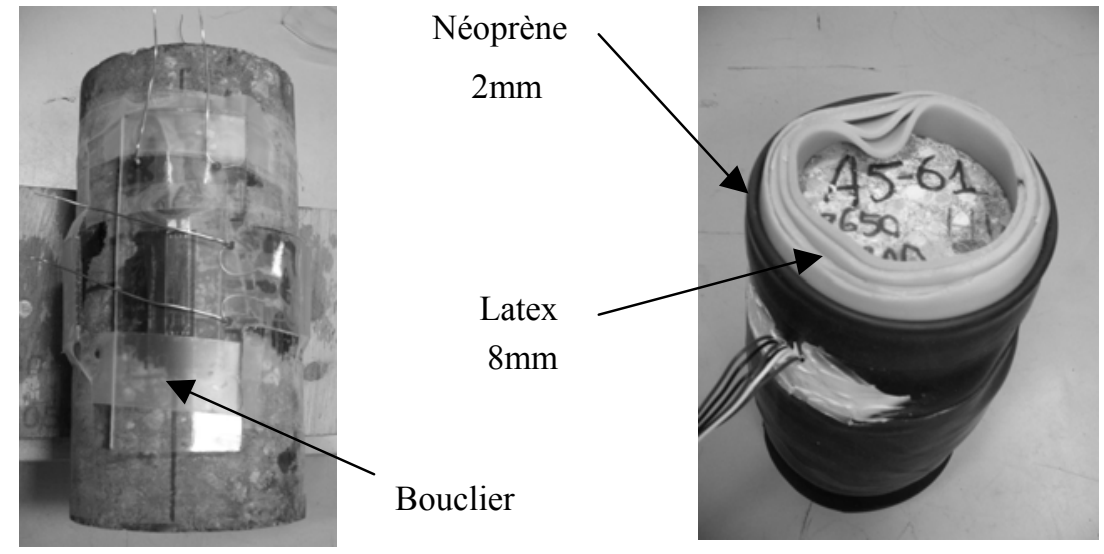

Figure 5. Dispositif de protection mis en place autour de l'échantillon : bouclier en plastique (photo de gauche) et membrane multicouches (photo de droite).

\subsection{Caractéristiques des échantillons de béton}

\section{Composition des bétons}

Afin d'étudier l'effet du rapport $\mathrm{E} / \mathrm{C}$ sur le comportement du béton, trois bétons avec différents rapport $\mathrm{E} / \mathrm{C}$ ont été formulés. La composition du béton de référence pour lequel une étude expérimentale exhaustive a été réalisée (Gabet et al., 2007) $(\mathrm{Vu}, 2007)$ est donnée dans la colonne EC06 du Tableau 1. Cette formulation correspond à celle d'un béton ordinaire en termes de résistance et d'affaissement. On peut cependant noter que la très bonne qualité du ciment utilisé, choisi pour mieux maitriser la reproductibilité du matériau, conduit à un volume de ciment particulièrement faible. Les granulats composés à $99 \%$ de quartzite sont issus de gisements naturels (granulats roulés). Leur taille maximale $(8 \mathrm{~mm})$ a été choisie au regard du diamètre de l'échantillon $(70 \mathrm{~mm})$.

A partir de la composition de ce béton de référence (béton EC06) dont le rapport $\mathrm{E} / \mathrm{C}$ est égal à 0,64 , deux bétons modifiés avec des rapports $\mathrm{E} / \mathrm{C}$ respectivement égaux à 0,4 (béton EC04) et à 0,8 (béton EC08) ont été formulés. Ces deux bétons modifiés ont un squelette granulaire et un volume de pâte de ciment très proches de ceux du béton de référence (Tableau 1). La quantité d'eau utilisée pour le béton EC04 étant faible, du super plastifiant "Sikafluid" est utilisé afin de lui conférer un affaissement de $7 \mathrm{~cm}$, équivalent à celui du béton EC06. Le béton EC08 possède en revanche un affaissement de $14 \mathrm{~cm}$, nettement supérieur aux deux autres bétons. 


\section{Confection des échantillons}

Une procédure de fabrication des éprouvettes de béton a été définie dans le but d'assurer une variabilité minimale des propriétés mécaniques du béton. Tous les bétons sont coulés dans un moule parallélépipédique par gâchées d'un volume de 13,5 litres. La mise en place des bétons EC06 et EC04 qui ont un affaissement identique est effectuée par vibration sur une table vibrante pendant une durée de 30s. La mise en place du béton EC08 est en revanche réalisée par simple piquage.

\begin{tabular}{|c|c|c|c|}
\hline Composition des bétons & EC04 & EC06 & EC08 \\
\hline Gravier D $0,5 / 8\left(\mathrm{~kg} / \mathrm{m}^{3}\right)$ & 1000 & 1008 & 991 \\
\hline Sable D $1800 \mu \mathrm{m}\left(\mathrm{kg} / \mathrm{m}^{3}\right)$ & 832 & 838 & 824 \\
\hline $\begin{array}{l}\text { Ciment CEM I 52,5 N PM ES CP2 } \\
\text { (Vicat) }\left(\mathrm{kg} / \mathrm{m}^{3}\right)\end{array}$ & 349 & 263 & 226 \\
\hline Eau & 136 & 169 & 181 \\
\hline Super plastifiant "Sikafluid"' $\left(\mathrm{kg} / \mathrm{m}^{3}\right)$ & 4,5 & 0 & 0 \\
\hline Densité $\left(\mathrm{kg} / \mathrm{m}^{3}\right)$ & 2322 & 2278 & 2252 \\
\hline \multicolumn{4}{|l|}{ Propriétés mécaniques des bétons } \\
\hline $\begin{array}{l}\text { Résistance moyenne testée en } \\
\text { compression simple à } 28 \text { jours }(\mathrm{MPa})\end{array}$ & $57^{(*)}$ & 28,6 & $21^{(*)}$ \\
\hline $\begin{array}{l}\text { Affaissement moyen mesuré au cône } \\
\text { d'Abrams }(\mathrm{cm})\end{array}$ & 7 & 6,9 & 14 \\
\hline $\begin{array}{l}\text { Volume d'air occlus mesuré dans les } \\
\text { bétons frais (par l'aéromètre) }\left(1 / \mathrm{m}^{3}\right)\end{array}$ & 41 & 34 & 50,5 \\
\hline Porosité accessible à l'eau (\%) & 7 & 12 & 14 \\
\hline Rapport E/C & 0,4 & 0,64 & 0,8 \\
\hline Volume de la pâte de ciment $\mathrm{V}_{\mathrm{p}}\left(\mathrm{m}^{3} / \mathrm{m}^{3}\right)$ & 0,250 & 0,252 & 0,248 \\
\hline
\end{tabular}

Tableau 1. Compositions et propriétés mécaniques des bétons EC04, EC06 et EC08; (*) Valeurs estimées à partir d'essais de compression simple réalisés à un âge $j>28$ jours.

Le bloc de béton, démoulé $24 \mathrm{~h}$ après le coulage, est ensuite conservé pendant 28 jours dans une ambiance saturée à l'intérieur de sacs plastiques eux-mêmes plongés dans l'eau afin d'isoler le béton d'un point de vue physique et thermique. Les échantillons de béton sont ensuite carottés, sciés puis rectifiés. Toutes ces étapes d'usinage sont effectuées sous une lubrification à l'eau pour éviter tout échauffement du béton. Le défaut de parallélisme des deux surfaces d'appui de l'échantillon est inférieur à $0,1 \mathrm{~mm}$ pour un diamètre $70 \mathrm{~mm}$. 


\section{Conservation des échantillons}

Après usinage, les échantillons sont conservés dans l'eau pendant trois mois selon une procédure de conservation identique pour tous les bétons. Les échantillons sont ensuite séchés dans une étuve à une température de $50^{\circ} \mathrm{C}$ et une humidité relative de $8 \%$. Tous les échantillons testés pour cette étude ont été conservés dans l'étuve pendant une durée de trois à six mois. Notons qu'après un mois de séchage dans l'étuve, la variation de masse journalière relative des échantillons ne dépassant plus $0,1 \%$, leur masse peut être considérée comme stabilisée.

Nous avons étudié l'évolution de la résistance à la rupture en compression simple des échantillons en fonction du temps de séchage dans l'étuve pour les trois bétons étudiés. Il s'avère que la variation de résistance, entre des échantillons conservés dans l'étuve 3 mois ou 6 mois, est inférieure à $12 \%$. Cet écart n'est guère supérieur à la dispersion des résultats de $7 \%$ mesurée sur des échantillons d'âge identique de béton EC06. Pour la suite de cet article l'effet de l'âge du béton sur les résultats d'essais triaxiaux est donc négligé. On considèrera que les résultats sont caractéristiques d'un béton de 8 mois d'âge conservé 4 mois dans l'eau à $20^{\circ} \mathrm{C}$ puis 4 mois dans une étuve à $50^{\circ} \mathrm{C}$.

\subsection{Trajet de chargement}

A l'exception des essais de compression simple, tous les essais triaxiaux ont été réalisés avec un trajet de chargement similaire de type compression triaxiale. L'essai débute par une phase hydrostatique, pendant laquelle la pression de confinement augmente à une vitesse de $1,67 \mathrm{MPa} / \mathrm{s}$ jusqu'à la pression souhaitée. La phase déviatoire est ensuite conduite, à pression de confinement constante, en imposant une vitesse de déplacement constante de $20 \mu \mathrm{m} / \mathrm{s}$ pour le vérin axial. Cela correspond à une vitesse de déformation de l'ordre de $10^{-4} / \mathrm{s}$ pour l'échantillon. La phase de décharge est symétrique par rapport à la phase de charge. La Figure 6 synthétise l'ensemble des trajets de chargement des essais réalisés pour cette étude.

Notons que les contraintes sont comptées positives en compression. On note $\sigma_{x}$ la contrainte axiale, $\mathrm{p}$ la pression de confinement, $\sigma_{m}$ la contrainte moyenne et q la contrainte déviatoire scalaire :

$$
\begin{gathered}
\sigma_{m}=\frac{\sigma_{x}+2 p}{3} \\
q=\sigma_{x}-p
\end{gathered}
$$

Les essais de compression simple ont été effectués sur une autre presse du laboratoire 3S-R. Ces essais sont pilotés en déplacement avec une vitesse de 
déformation de l'ordre de $10^{-5} / \mathrm{s}$. Les échantillons ont les mêmes dimensions que ceux utilisés pour les essais triaxiaux. Le récapitulatif des essais effectués est présenté dans le Tableau 2.

\section{Résultats des essais}

On montre dans ce paragraphe les résultats des essais de compression simple qui permettent de caractériser et de comparer le comportement uniaxial des trois bétons étudiés. Les résultats des essais triaxiaux sont ensuite présentés en termes de contrainte axiale en fonction des composantes de la déformation, séparément pour chacun des bétons.

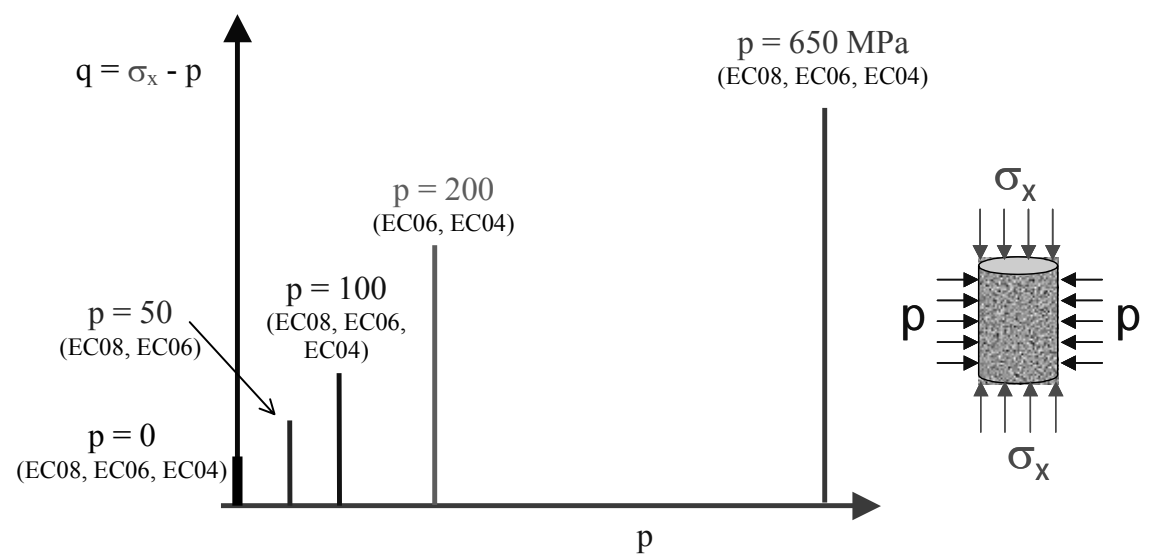

Figure 6. Chemin de chargement des essais réalisés : contrainte déviatoire q en fonction de la pression de confinement $p$ avec $\sigma_{x}$ : contrainte axiale ; EC08 : $E / C=0,8 ; E C 06: E / C=0,64 ; E C 04: E / C=0,4$. 


\begin{tabular}{|c|l|c|}
\hline Béton & $\begin{array}{c}\text { Pression de confinement } \\
\mathrm{p}(\mathrm{MPa})\end{array}$ & Numéro de l'échantillon \\
\hline & $\mathrm{p}=0$ & EC04-3 \\
$\mathrm{EC04}$ & $\mathrm{p}=100$ & EC04-1 \\
$\mathrm{E} / \mathrm{C}=0,4$ & $\mathrm{p}=200$ & $\mathrm{EC04-4}$ \\
& $\mathrm{p}=650(\mathrm{n} 1)$ & $\mathrm{EC04-5}$ \\
& $\mathrm{p}=650(\mathrm{n} 2)$ & $\mathrm{EC04-8}$ \\
\hline & $\mathrm{p}=0$ & $\mathrm{~A} 0-8$ \\
$\mathrm{EC06}$ & $\mathrm{p}=50$ & $\mathrm{~A} 0-5$ \\
$\mathrm{E} / \mathrm{C}=0,64$ & $\mathrm{p}=100\left(^{*}\right)$ & $\mathrm{A} 9-75$ \\
& $\mathrm{p}=200\left(^{*}\right)$ & $\mathrm{A} 9-76$ \\
& $\mathrm{p}=650$ & $\mathrm{~A} 11-5$ \\
\hline & $\mathrm{p}=0$ & $\mathrm{EC08-4}$ \\
$\mathrm{EC} 08$ & $\mathrm{p}=50$ & $\mathrm{EC08-2}$ \\
$\mathrm{E} / \mathrm{C}=0,8$ & $\mathrm{p}=100$ & $\mathrm{EC08-9}$ \\
& $\mathrm{p}=650$ & $\mathrm{EC08-1}$ \\
\hline
\end{tabular}

Tableau 2. Récapitulatif des essais effectués en fonction du type de béton et de la pression de confinement ; $\left(^{*}\right)$ Essais réalisés par T. Gabet (Gabet et al., 2007).

\subsection{Essais de compression simple}

La Figure 7 présente les résultats des essais de compression simple réalisés sur les trois types de béton. La Figure 7a montre l'évolution de la contrainte axiale en fonction des composantes de la déformation. Comme cela est attendu, on observe une augmentation du module d'Young $\mathrm{E}$ et de la contrainte à la rupture $\sigma_{\max } \mathrm{du}$ béton avec la diminution du rapport $\mathrm{E} / \mathrm{C}$. Les valeurs numériques de $\mathrm{E}$, de $\sigma_{\max }$ et du coefficient de Poisson $v$ obtenues pour les trois bétons sont indiquées dans le Tableau 3. Conformément à ce qui est écrit dans littérature (ACI 363R-92, 1994), (ACI 318-89, 1996), on note que l'augmentation du module d'Young est, en proportion, inférieure à celle de l'augmentation de la résistance du béton. La Figure $7 \mathrm{~b}$ montre également que le béton EC04 a un comportement du type élastiquefragile tandis que les bétons EC06 et EC08 ont un comportement nettement plus ductile de type adoucissant. Ces résultats sont conformes à ce que l'on peut attendre compte tenu des valeurs des rapports E/C.

La Figure $7 \mathrm{~b}$ montre l'évolution de la contrainte moyenne en fonction de la déformation volumique du béton. Durant la phase contractante de l'essai, on constate que pour une contrainte moyenne donnée, la déformation volumique du béton est d'autant plus faible que le rapport $\mathrm{E} / \mathrm{C}$ est faible. Le squelette granulaire des trois bétons ayant la même composition, la porosité du béton augmente avec E/C. Ce résultat est aussi parfaitement concordant avec l'évolution du module d'Young et du coefficient de Poisson observées dans le Tableau 3. 


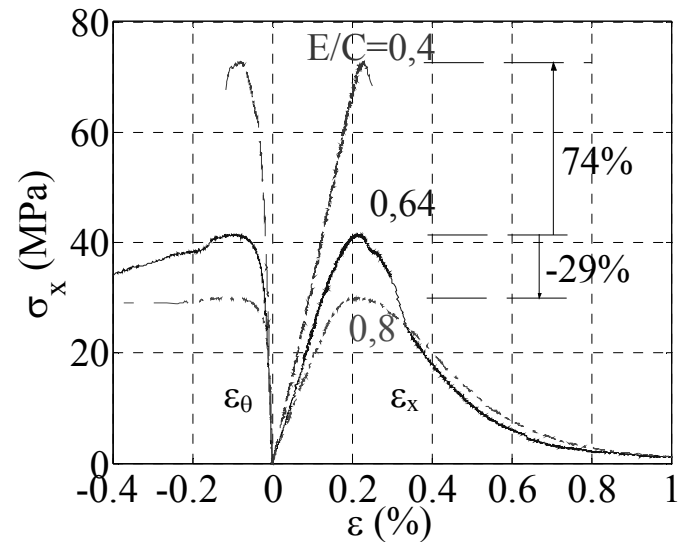

(a)

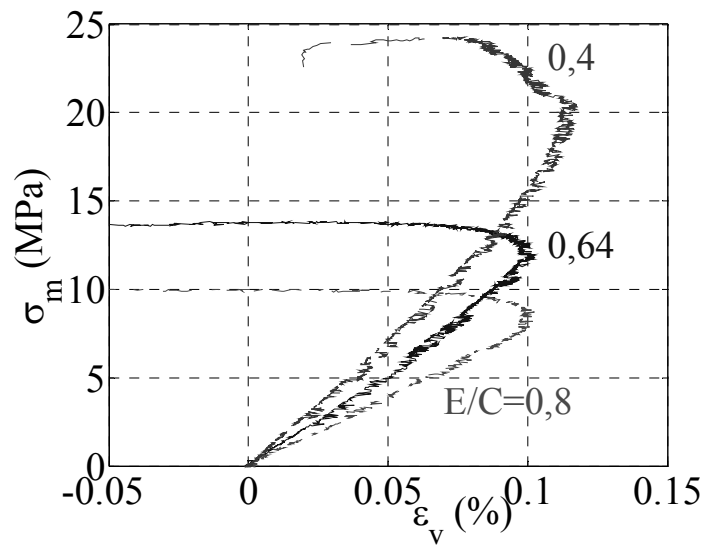

(b)

Figure 7. Essais de compression simple sur les bétons EC08, EC06 et EC04 : (a) Contrainte axiale $\sigma_{x}$ en fonction des composantes de la déformation $\varepsilon_{x}$ et $\varepsilon_{\theta}$; (b) Contrainte moyenne $\sigma_{m}$ en fonction de la déformation volumique $\varepsilon_{v}$

\begin{tabular}{|l|c|c|c|}
\hline Béton & EC08 & EC06 & EC04 \\
\hline Rapport E/C du béton & 0,8 & 0,64 & 0,4 \\
\hline Contrainte à la rupture $\left(\sigma_{\max }\right)$ & $30 \mathrm{MPa}$ & $42 \mathrm{MPa}$ & $73 \mathrm{MPa}$ \\
\hline Module de Young $(\mathrm{E})$ & $19 \mathrm{GPa}$ & $24 \mathrm{GPa}$ & $33 \mathrm{Gpa}$ \\
\hline Coefficient de Poisson $(v)$ & 0,12 & 0,14 & 0,15 \\
\hline
\end{tabular}

Tableau 3. Essais de compression simple: identification des principales caractéristiques des bétons. 


\subsection{Essais triaxiaux}

Les Figure 8,Figure 9 et Figure 10 montrent l'évolution de la contrainte axiale en fonction des composantes de la déformation pour les essais triaxiaux à différentes pressions de confinement. Chaque figure correspond à une composition de béton donnée. Les échelles des trois figures sont identiques.

Pour un type de béton donné, durant la phase hydrostatique (Figure 8b,Figure $9 \mathrm{~b}$ et Figure 10b) on observe que les essais suivent la même courbe quelle que soit la pression de confinement. Cela montre une bonne reproductibilité du comportement des bétons et confirme la pertinence de la procédure de préparation des échantillons. Durant cette même phase, les courbes de déformation axiale et circonférentielle sont très proches. Le comportement des bétons est donc quasiment isotrope pour ces niveaux de sollicitation. Pour les bétons EC06 et EC08 (Figure 8b etFigure 9b) on peut noter que les courbes $\sigma(\varepsilon)$ sous sollicitation hydrostatique ont une forme en « $\mathrm{s}$ ». Après une phase linéaire assez courte (jusqu'à 40 à $60 \mathrm{MPa}$ de confinement), on observe une diminution de la raideur tangente de ces bétons puis un raidissement au-delà de 200 à $300 \mathrm{MPa}$ de confinement. La diminution de la raideur peut être attribuée à un endommagement de la matrice cimentaire au-delà de la phase élastique et le raidissement à une densification du matériau à mesure que la porosité du béton se referme lorsque la déformation hydrostatique devient significative. Pour le béton EC04 (Figure 10b) on observe une phase linéaire beaucoup plus longue (jusqu'à $200 \mathrm{MPa}$ de confinement) suivie d'une diminution de la raideur qui devient ensuite constante. Le niveau de confinement atteint semble insuffisant pour que le raidissement du béton soit perceptible.

Concernant la phase déviatoire du comportement (Figure 8a,Figure 9a et Figure 10a), quel que soit le type de béton considéré, on observe que le confinement augmente très fortement à la fois la ductilité et la capacité de chargement du béton. Par ailleurs, pour les niveaux de confinement les plus importants l'écrouissage positif du matériau est tel qu'aucun pic de contrainte n'est atteint avant le déchargement. La comparaison des trois bétons montre que la variation du rapport E/C modifie le seuil de confinement qui correspond à la transition fragile-ductile du comportement déviatoire. On constate par exemple que pour un niveau de confinement de $50 \mathrm{MPa}$, le béton EC06 présente un comportement adoucissant avec un pic de contrainte nettement observable tandis que le béton $\mathrm{EC} 08$ présente déjà un comportement de type parfaitement plastique (plateau horizontal). De la même manière, pour un niveau de confinement de $100 \mathrm{MPa}$, le béton EC04 présente un pic de contrainte alors que les bétons EC06 et EC08 montrent un plateau horizontal. 


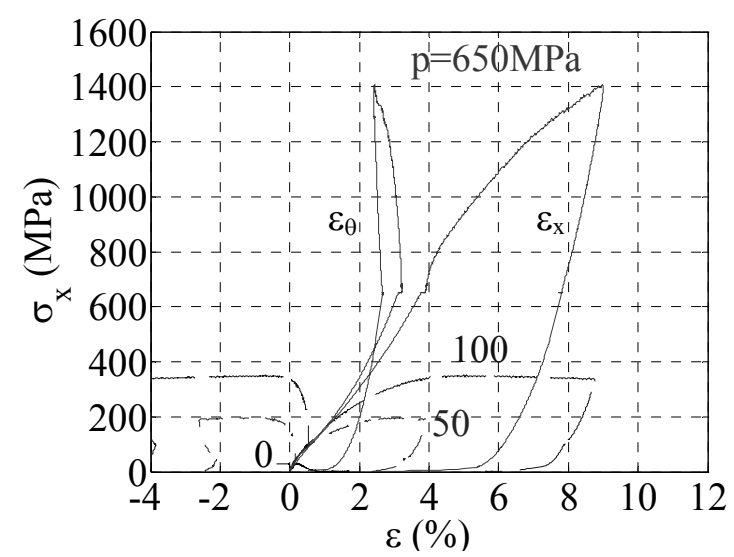

(a) $E / C=0,8$

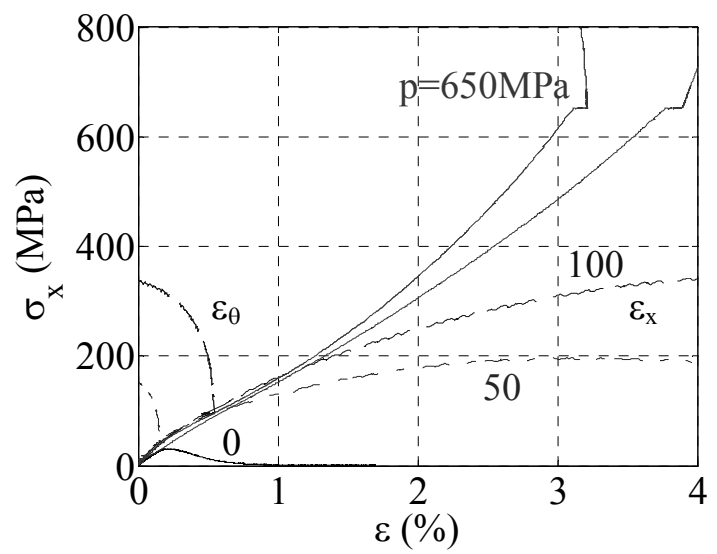

(b) $E / C=0,8$ - zoom sur les phases hydrostatiques du chargement

Figure 8. Essais triaxiaux à différentes pressions de confinement $p$ sur béton EC08 $(E / C=0,8)$ : Contrainte axiale $\sigma_{x}$ en fonction des composantes de la déformation $\varepsilon_{X}$ et $\varepsilon_{\theta}$ 
16 Revue. Volume $\mathrm{X}-\mathrm{n}^{\circ} \mathrm{x} /$ année

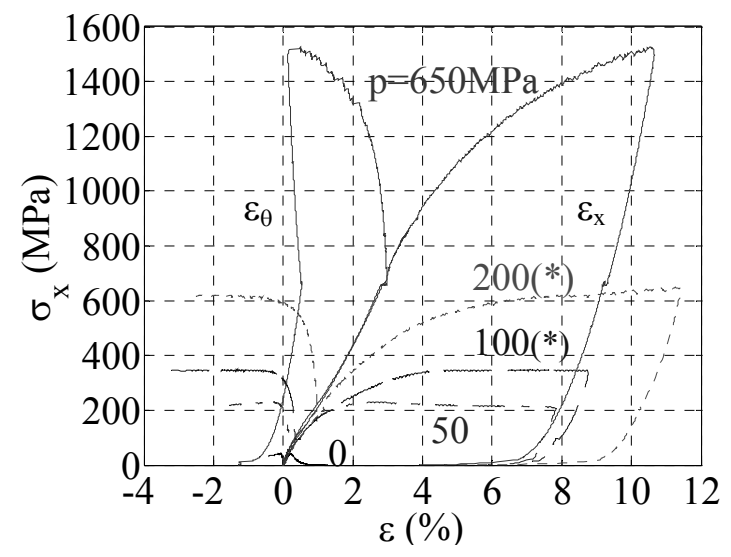

(a) $E / C=0,64$

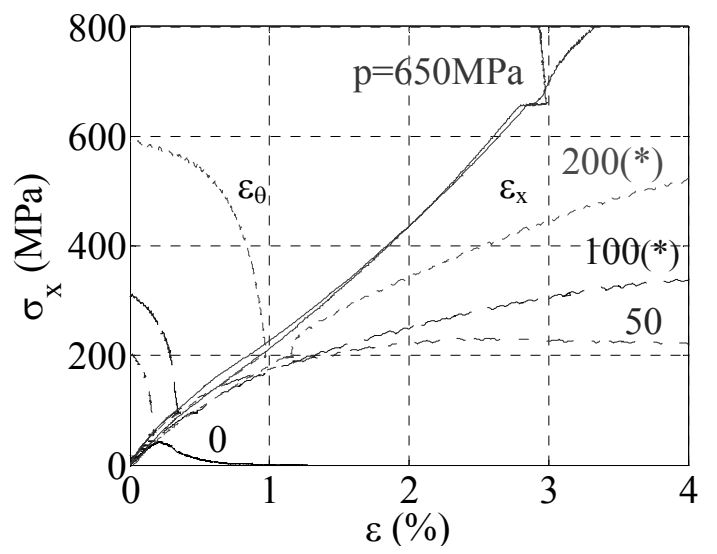

(b) $E / C=0,64-$ zoom sur les phases hydrostatiques du chargement

Figure 9. Essais triaxiaux à différentes pressions de confinement p sur béton EC08 $(E / C=0,8)$ : Contrainte axiale $\sigma_{x}$ en fonction des composantes de la déformation $\varepsilon_{x}$ et $\varepsilon_{\theta} ;\left(^{*}\right)$ Essais réalisés par T. Gabet (Gabet et al., 2007). 


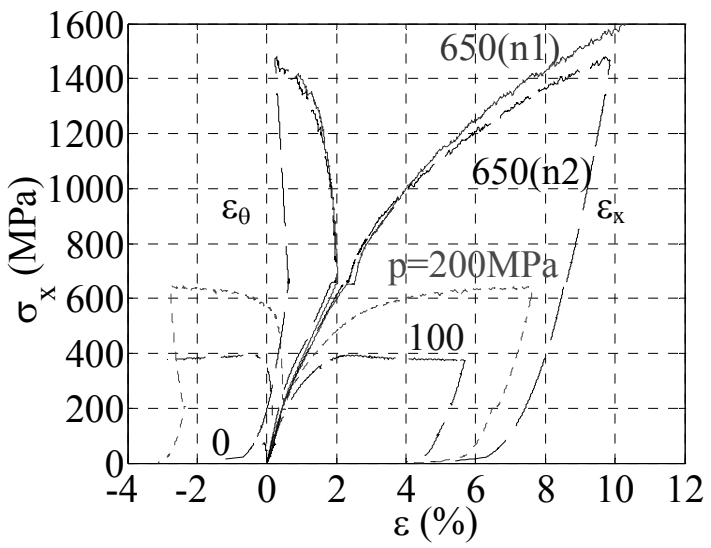

(a) $E / C=0,4$

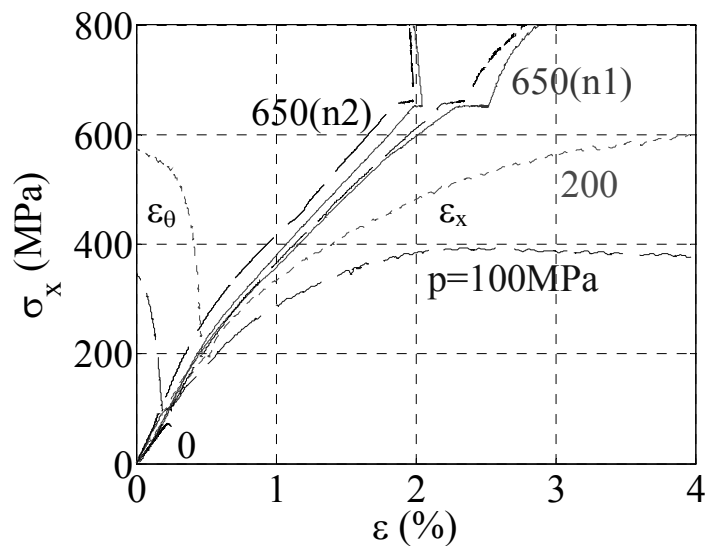

(b) $E / C=0,4$ - zoom sur les phases hydrostatiques du chargement

Figure 10. Essais triaxiaux à différentes pressions de confinement $p$ sur béton EC08 $(E / C=0,8)$ : Contrainte axiale $\sigma_{x}$ en fonction des composantes de la déformation $\varepsilon_{x}$ et $\varepsilon_{\theta}$

\section{Comportement volumique et comportement déviatoire}

Afin d'évaluer de manière plus précise l'effet du rapport E/C sur le comportement du béton, on compare dans ce paragraphe les courbes de comportement volumique et de comportement déviatoire des trois bétons étudiés à différents niveaux de confinement. La contrainte moyenne, la déformation volumique, et la contrainte déviatoire sont estimées à partir des mesures présentées au paragraphe précédent. 


\section{Essais à 50 MPa de confinement}

La Figure 11 présente les courbes de comportement volumique (Figure 11a) et déviatoire (Figure 11b) des bétons EC06 et EC08 testés à $50 \mathrm{MPa}$ de confinement. La déformation volumique est quasiment linéaire dans la phase hydrostatique pour les deux bétons. On constate que la déformation volumique du béton EC08 est plus importante que celle du béton EC06, en particulier dans la phase déviatoire de l'essai. La porosité du béton EC08 étant plus importante que celle du béton EC06, ce résultat était attendu. Ce résultat est aussi cohérent avec celui obtenu lors d'essais triaxiaux à $15 \mathrm{MPa}$ de confinement sur des mortiers (Yurtdas, 2005).

On observe par ailleurs sur la Figure $11 \mathrm{~b}$ que la valeur de la contrainte déviatoire maximale du béton EC06 est de 13\% plus grande celle du béton EC08. Notons qu'en compression simple, la différence entre les résistances en compression simple des deux bétons est d'environ 38\%. L'écart de résistance relatif entre les deux bétons est donc nettement plus faible sous l'effet du confinement latéral.

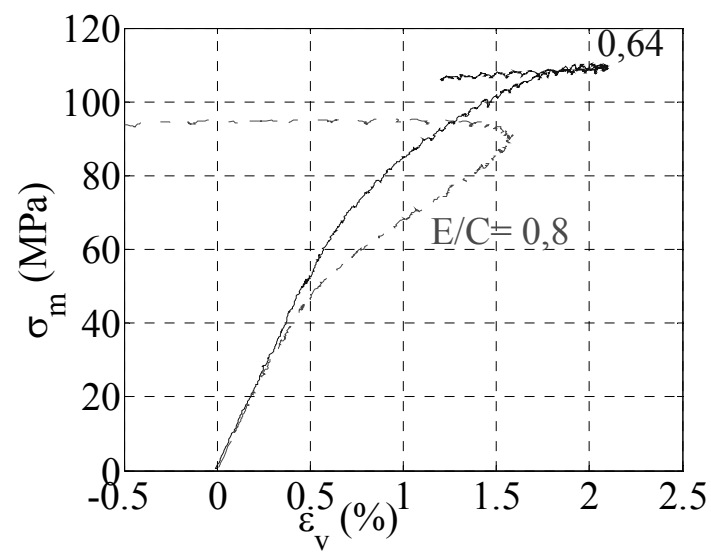

(a) 


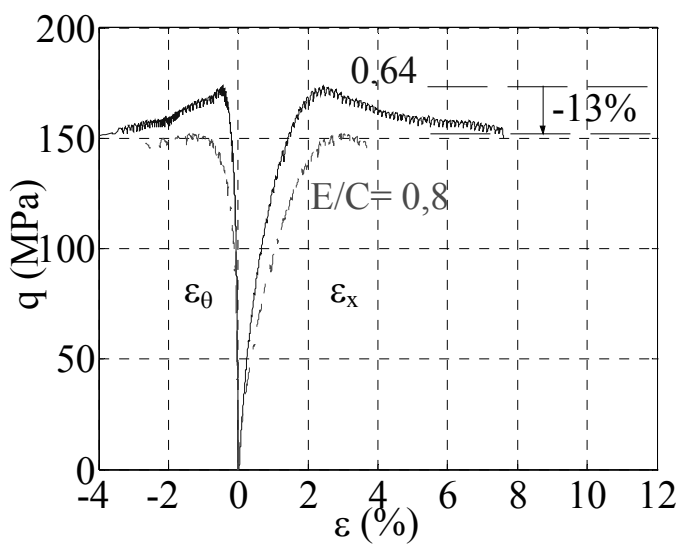

(b)

Figure 11. Essais triaxiaux à $50 \mathrm{MPa}$ de confinement pour les bétons EC06 et EC08 : (a) Contrainte moyenne $\sigma_{m}$ en fonction de déformation volumique $\varepsilon_{v}$; (b) Déviateur de contrainte $q$ en fonction des composantes de la déformation $\varepsilon_{x}$ et $\varepsilon_{\theta}$

\section{Essais à 100 MPa de confinement}

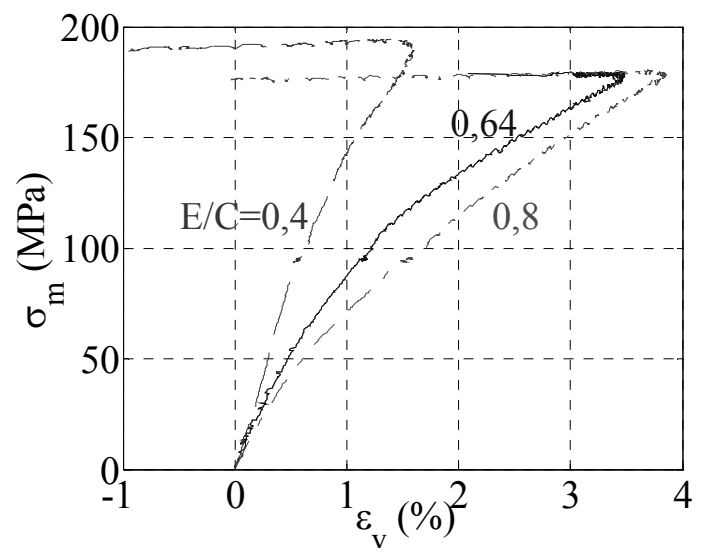

(a) 


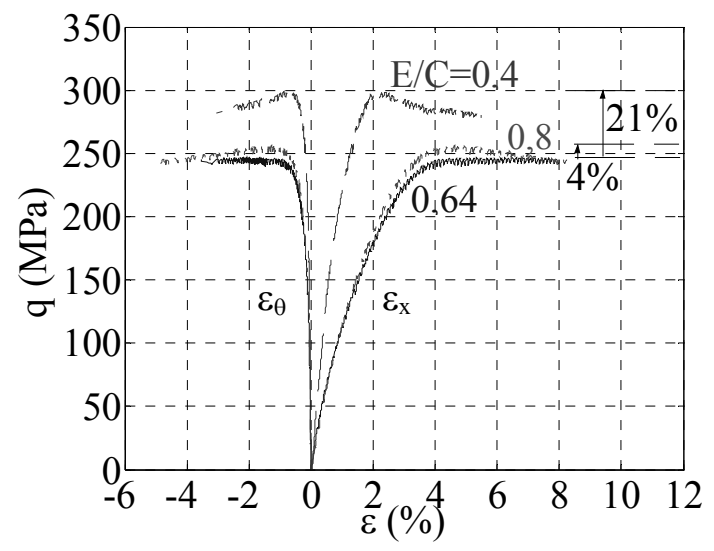

(b)

Figure 12. Essais triaxiaux à $100 \mathrm{MPa}$ de confinement pour les bétons EC04, EC06 et EC08: (a) Contrainte moyenne $\sigma_{m}$ en fonction de déformation volumique $\varepsilon_{v}$; (b) Déviateur de contrainte $q$ en fonction des composantes de la déformation $\varepsilon_{x}$ et $\varepsilon_{\theta}$

La Figure 12 présente les courbes de comportement volumique (Figure 12a) et déviatoire (Figure 12b) des bétons EC08, EC06 et EC04 testés à $100 \mathrm{MPa}$ de confinement. La Figure 12 montre que la déformation volumique du béton EC06 est moins élevée que celle du béton EC04 mais plus importante que celle du béton EC08. Ce résultat attendu s'explique par les écarts de porosité entre les trois bétons. On remarque par ailleurs que le comportement hydrostatique du béton EC04 est encore linéaire à $100 \mathrm{MPa}$ tandis que les deux autres bétons montrent une diminution importante de leur module de compressibilité tangent. La matrice cimentaire du béton EC04 se comporte donc de manière quasiment élastique pour un tel confinement tandis que celles dont le rapport E/C est plus important sont déjà fortement endommagées.

La Figure 12b montre que les courbes de comportement déviatoire des bétons EC08 et EC06 sont pratiquement confondues. On observe un écart de seulement 4\% entre les valeurs de la contrainte au pic pour les deux bétons ce qui n'est pas significatif par rapport à la dispersion possible. Ce résultat peut s'expliquer par le fait que la cohésion de la matrice cimentaire de ces deux bétons est fortement endommagée pendant la phase hydrostatique. A la fin de cette phase, le béton se comporte ainsi comme un empilement granulaire quasiment non-cohésif. La réponse déviatoire du béton est donc fortement liée à son empilement granulaire et non plus à la cohésion de la matrice cimentaire. Or les bétons étudiés ont un squelette granulaire identique (rapport Gravier/Sable et volume de granulats identiques). Seule la quantité de ciment est légèrement différente entre les compositions EC06 et EC08 (écart de $42 \mathrm{~kg} / \mathrm{m}^{3}$ ). Si l'on suppose que le ciment est entièrement hydraté, 
cela correspond à une différence sur le volume des hydrates qui représente moins de $3 \%$ du volume total du béton. Les empilements granulaires des bétons EC06 et EC08 sont donc très similaires et leur faible différence n'est pas perceptible sur le comportement déviatoire du béton au-delà de $100 \mathrm{MPa}$ de confinement.

La Figure 12b montre aussi que la raideur tangente du béton EC04 est beaucoup plus grande que celles des bétons EC06 et EC08. En outre, le comportement du béton EC04 est encore de type fragile et la valeur la contrainte au pic est de $21 \%$ plus grande que celle du béton EC06. Pour ce confinement, la matrice cimentaire du béton EC04 présente encore une cohésion importante (comportement volumique élastique) qui joue un rôle important dans la réponse déviatoire de ce béton.

\section{Essais à 200 MPa de confinement}

La Figure 13 présente les courbes de comportement volumique (Figure 13a) et déviatoire (Figure 13b) des bétons EC06 et EC04 testés à $200 \mathrm{MPa}$ de confinement. De même que pour des niveaux de confinement moins importants, la Figure 13a met en évidence que la déformation volumique du béton EC06 est nettement plus importante que celle du béton EC04. Cet effet s'explique aisément par la différence de porosité entre la matrice cimentaire des deux bétons.

La Figure 13b montre que la raideur du béton EC04 est plus grande que celle du béton EC06, mais qu'elle l'est faiblement par rapport à ce qui était observé à plus faible confinement. De la même manière, on constate que la valeur du déviateur atteint pour le béton EC04 n'est que de 9\% supérieure à celle du béton EC06 ce qui est peu par rapport à l'écart observé à $100 \mathrm{MPa}$ de confinement. Ces observations révèlent que pour un confinement passant de 100 à $200 \mathrm{MPa}$, la matrice cimentaire $\mathrm{du}$ béton EC04 est progressivement endommagée. Cet endommagement est d'ailleurs mis en évidence par la non-linéarité observée au-delà de $150 \mathrm{MPa}$ sur le comportement hydrostatique (Figure 13a). L'écart entre le comportement des deux bétons montre cependant que la matrice du béton EC04 n'est pas totalement détruite et présente encore une cohésion significative sous une pression de confinement de $200 \mathrm{MPa}$. 


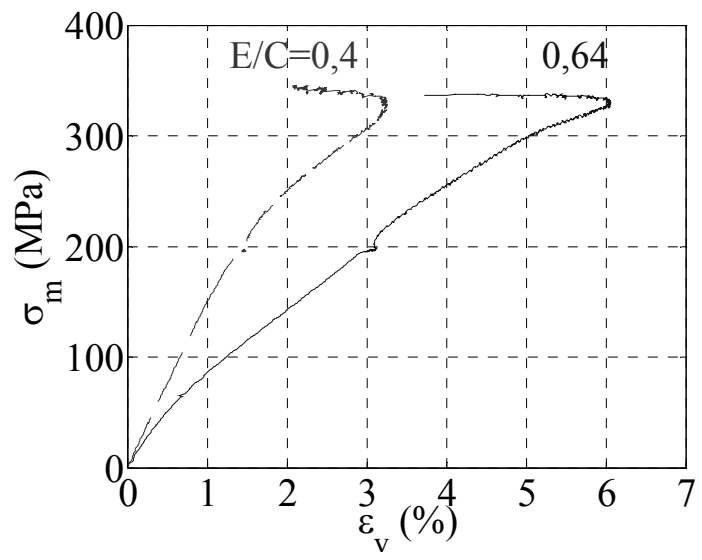

(a)

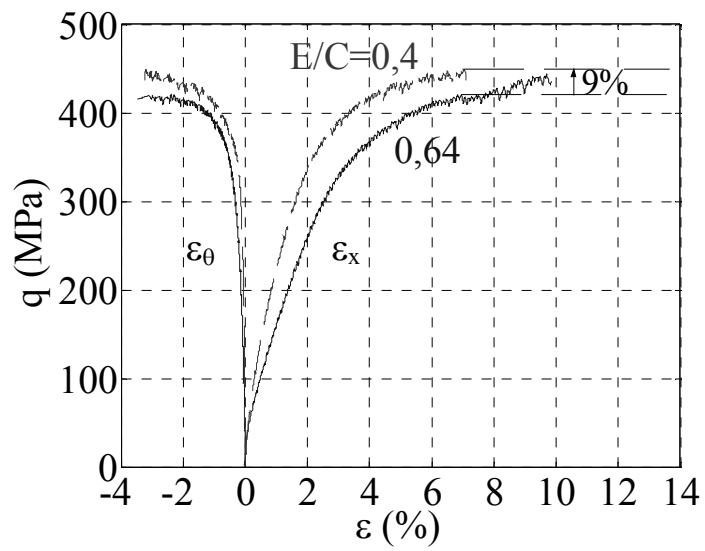

(b)

Figure 13. Essais triaxiaux à $200 \mathrm{MPa}$ de confinement pour les bétons EC04 et EC06 : (a) Contrainte moyenne $\sigma_{m}$ en fonction de déformation volumique $\varepsilon_{v}$; (b) Déviateur de contrainte $q$ en fonction des composantes de la déformation $\varepsilon_{x}$ et $\varepsilon_{\theta}$

\section{Essais à 650 MPa de confinement}

La Figure 14 présente les courbes de comportement volumique (Figure 14a) et déviatoire (Figure 14b) des bétons EC08, EC06 et EC04 testés à $650 \mathrm{MPa}$ de confinement. On observe que les courbes obtenues pour les deux échantillons EC04(n1) et EC04(n2) sont très proches ce qui confirme la faible dispersion des résultats d'essais. 
La comparaison du comportement volumique des trois bétons sur la Figure 14a montre une nouvelle fois que la déformation volumique du béton est accrue avec l'augmentation du rapport E/C. Sur le plan quantitatif on peut remarquer que l'écart entre les courbes EC06 et EC04 est environ deux fois plus important que celui observé entre les courbes EC06 et EC08. Or l'écart entre les porosités des bétons EC06 et EC04 est lui-même environ deux fois plus grand que l'écart entre les porosités des bétons EC06 et EC08. On a donc au premier ordre une corrélation linéaire entre la variation de porosité du béton et sa variation de déformation volumique pour un squelette granulaire donné.

La Figure 14a montre enfin qu'au-delà $400 \mathrm{MPa}$ de confinement les courbes de comportement volumique des trois bétons semblent parallèles. Cela signifie que l'écart entre les déformations volumiques incrémentales des trois bétons n'est significatif qu'à faible confinement. Typiquement entre 0 et $200 \mathrm{MPa}$ si l'on compare les bétons EC06 et EC08 et entre 0 et $400 \mathrm{MPa}$ si l'on compare le béton EC04 aux deux autres.

Par ailleurs, on observe sur la Figure 14b que les courbes de comportement déviatoire des bétons EC08, EC06 et EC04 sont pratiquement confondues. Ce résultat confirme que sous fort confinement, la matrice cimentaire du béton perd sa cohésion. Le comportement du béton est alors entièrement gouverné par l'empilement granulaire qu'est devenu le béton. Les squelettes granulaires des bétons étudiés étant identiques, cela conduit à un comportement déviatoire similaire pour les trois bétons étudiés. Ainsi, au premier ordre, sous fort confinement, le comportement du béton devient insensible au rapport E/C.

Une observation plus fine des courbes sur la Figure $14 \mathrm{~b}$ montre tout de même que pour des niveaux de déviateur supérieurs à $600 \mathrm{MPa}$, les courbes $\left(\mathrm{q}, \varepsilon_{\mathrm{x}}\right)$ des échantillons de béton EC04 sont légèrement plus raides que celle observée pour le béton EC06. Cette petite différence de comportement s'explique peut-être par la différence entre les volumes d'hydrates des trois bétons. Le début de la phase déviatoire des essais favorise un réarrangement des grains au sein du béton qui se caractérise au niveau macroscopique par une augmentation de la déformation volumique par rapport à un essai hydrostatique à contrainte moyenne équivalente (phase déviatoire de la Figure 14a). On peut donc supposer qu'à faible niveau de contrainte déviatoire, la faible différence de volume de fines provenant de la destruction des hydrates ne joue pas de rôle significatif sur le comportement de l'empilement granulaire. En revanche, à des niveaux plus élevés, à mesure que la densité du matériau augmente et que la porosité diminue très fortement, il est plausible que la différence de volume de fines entre les trois bétons devienne perceptible. 


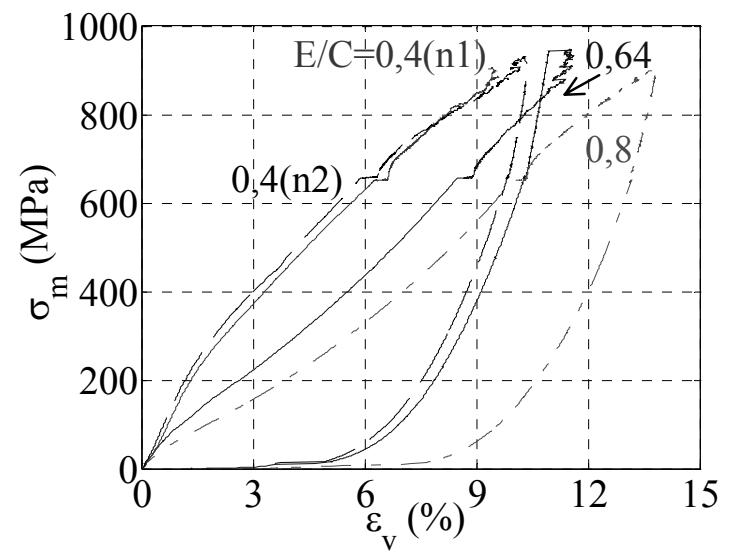

(a)

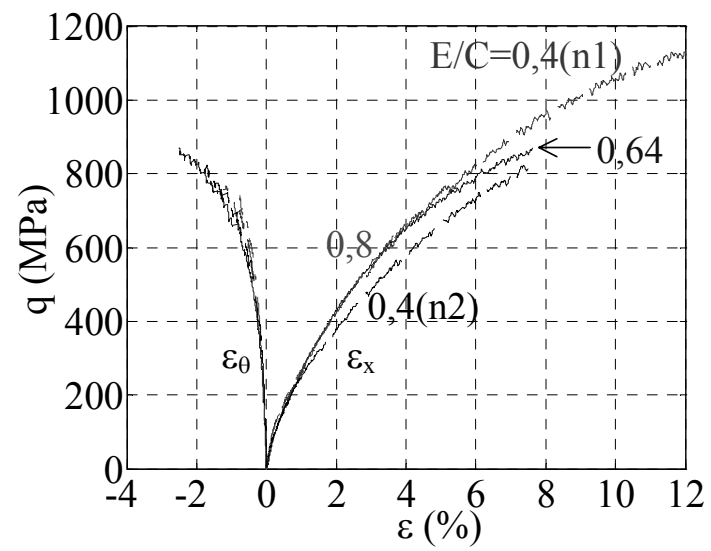

(b)

Figure 14. Essais triaxiaux à $650 \mathrm{MPa}$ de confinement pour les bétons EC04, EC06 et EC08: (a) Contrainte moyenne $\sigma_{m}$ en fonction de déformation volumique $\varepsilon_{v}$; (b) Déviateur de contrainte $q$ en fonction des composantes de la déformation $\varepsilon_{x}$ et $\varepsilon_{\theta}$

\section{Etats-limites}

Lors de la caractérisation expérimentale du comportement d'un matériau, différents critères peuvent être utilisés pour identifier sa courbe d'état-limite. Le critère le plus couramment utilisé est défini comme l'état de contrainte associé à la contrainte déviatoire maximale que le matériau peut supporter (état-limite en contrainte). Pour les géomatériaux qui ont un comportement dilatant à la rupture, cet 
état-limite en contrainte est souvent proche de l'état de contrainte associé au point de transition de la contractance du matériau à sa dilatance. Cet état correspond à l'état de déformation volumique maximale en termes de contraction que le matériau peut atteindre. Pour la suite nous définirons cet état de contrainte comme l'étatlimite en déformation du béton.

Les résultats des essais présentés aux paragraphes 3 et 4 montrent que pour les échantillons testés à fort confinement, le dispositif expérimental utilisé ne permet plus d'atteindre la valeur maximale de la contrainte avant le déchargement de l'essai. Il n'est donc plus possible de caractériser l'état-limite en contrainte du béton pour les forts niveaux de confinement. Seul l'état-limite en déformation peut être identifié pour tous les échantillons testés. Cette transition est estimée à partir des courbes de comportement volumique des échantillons testés (Figure 7a, Figure 11a, Figure 12a, Figure 13a et Figure 14a). Ces figures montrent que pour les faibles confinements, tant que le béton se comporte de manière « fragile », l'état-limite en déformation coïncide pratiquement avec l'état-limite en contrainte du béton. Ainsi, l'état-limite en déformation est choisi comme état-limite de référence dans cette étude.

La Figure 15a synthétise les états-limites en déformation des bétons EC08, EC06 et EC04 dans le plan déviatoire $\left(\sigma_{\mathrm{m}} ; \mathrm{q}\right)$ pour tous les essais réalisés. On observe que la capacité de chargement du béton croit fortement et de manière quasi-linéaire avec l'augmentation de la contrainte moyenne.

La Figure $15 \mathrm{~b}$ montre ces mêmes états limites dans un plan $\left(\sigma_{\mathrm{m}}, \mathrm{q} / \mathrm{q}_{\mathrm{EC} 06}\right)$ où $\mathrm{q}_{\mathrm{EC} 06}$ est le déviateur limite obtenu pour le béton de référence EC06 pour une pression de confinement identique. Cette présentation en termes de déviateur relatif à celui du béton de référence permet de mieux percevoir les différences entre les trois bétons. Cette figure permet de vérifier que pour de faibles niveaux de contrainte moyenne, l'état limite du béton est fortement dépendant du rapport E/C, c'est-à-dire de la résistance de la matrice cimentaire. Ce résultat était évidemment attendu. En revanche, on observe sur cette même figure que cette dépendance de l'état-limite du béton vis-à-vis du rapport $\mathrm{E} / \mathrm{C}$ diminue rapidement à mesure que la contrainte moyenne augmente. Ainsi, au-delà d'une contrainte moyenne critique $\sigma_{\mathrm{mc}}$ la courbe d'état-limite devient indépendante du rapport $\mathrm{E} / \mathrm{C}$.

Cette contrainte moyenne critique dépend elle-même du rapport E/C, elle augmente lorsque $\mathrm{E} / \mathrm{C}$ diminue. C'est-à-dire que la contrainte moyenne, au-delà de laquelle le rapport $\mathrm{E} / \mathrm{C}$ n'a plus d'influence, est d'autant plus grande que la résistance de la matrice cimentaire est importante. D'après la Figure 15b, lorsque E/C diminue de 0,64 à 0,4 alors $\sigma_{\mathrm{mc}}$ augmente de $180 \mathrm{MPa}$ à $330 \mathrm{MPa}$. 


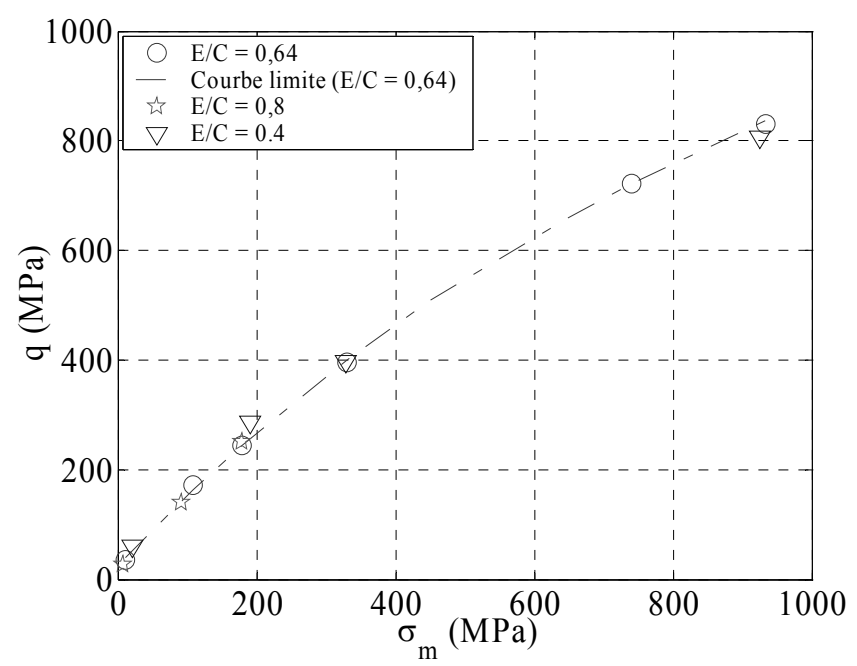

(a)

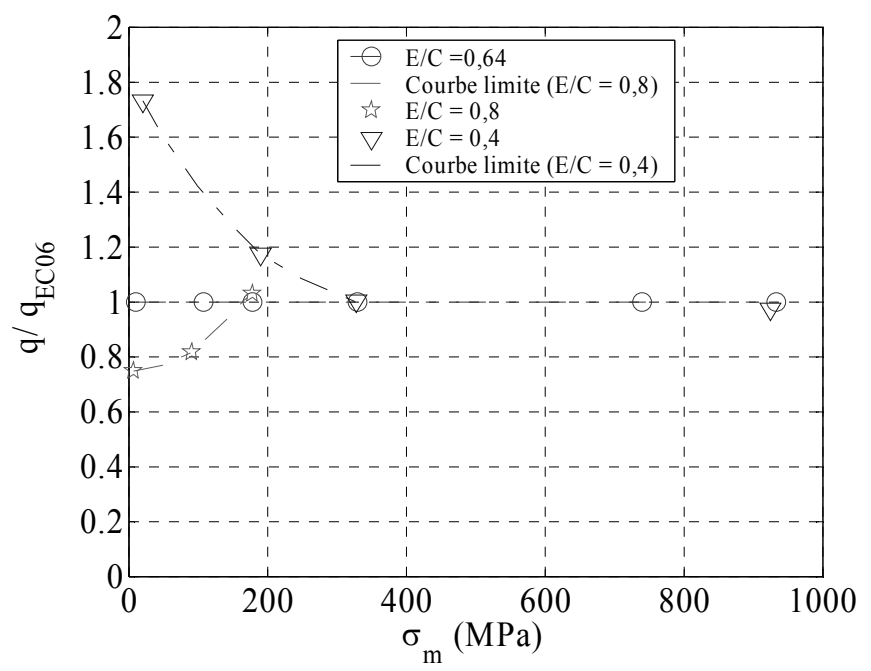

(b)

Figure 15. Etats limites des bétons EC04, EC06 et EC06 : (a) Contrainte déviatoire $q$ en fonction de la contrainte moyenne $\sigma_{m}$; (b) Déviateur relatif $q / q_{E C 06}$ en fonction de la contrainte moyenne $\sigma_{m}$ où $q_{E C 06}$ est la contrainte déviatoire associée à l'état limite du béton de référence EC06.

L'interprétation des résultats ci-dessus peut se faire de la manière suivante. A faible niveau de contrainte moyenne $\left(\sigma_{\mathrm{m}}<<\sigma_{\mathrm{mc}}\right)$ le comportement du béton est en 
grande partie gouverné par la cohésion de la matrice cimentaire. La transition contractance-dilatance est associée à l'ouverture de microfissures au sein de la structure du matériau lors de l'endommagement progressif de la matrice cimentaire (Mazars, 1984). Pour un même niveau de contrainte moyenne, plus le rapport E/C du béton est faible, plus la cohésion de la matrice cimentaire est importante et plus la résistance du béton est élevée.

Lorsque le niveau de contrainte moyenne augmente $\left(\sigma_{\mathrm{m}} \approx \sigma_{\mathrm{mc}}\right)$, la différence relative entre la cohésion résiduelle des différents bétons devient plus faible, ce qui conduit à une diminution des écarts entre les déviateurs atteints. A fort niveau de contrainte moyenne $\left(\sigma_{\mathrm{m}}>\sigma_{\mathrm{mc}}\right)$, la cohésion de la matrice cimentaire des bétons est complètement détruite. Les bétons se comportent alors comme des empilements granulaires. L'état limite en déformation du béton correspond à la compacité maximale que cet empilement peut atteindre. Les squelettes granulaires des bétons étudiés étant identiques, les empilements associés sont très proches. Ainsi au-delà de la contrainte moyenne critique les trois bétons se comportent de manière identique quel que soit leur rapport $\mathrm{E} / \mathrm{C}$.

\section{Conclusion}

Le contexte de cette étude concerne l'identification du comportement du béton sous sollicitations extrêmes. Pour reproduire de forts niveaux de contrainte avec des trajets de chargement bien contrôlés, des essais statiques ont été réalisés sur des échantillons de béton en utilisant une presse triaxiale de très grande capacité. Les résultats des essais présentés dans cet article concernent plus spécifiquement l'effet $\mathrm{du}$ rapport $\mathrm{E} / \mathrm{C}$, entrant dans la composition du béton, sur son comportement sous fort confinement. Ainsi, à partir d'un béton de référence ordinaire $(\mathrm{E} / \mathrm{C}=0,6)$, deux autres bétons avec des rapports $\mathrm{E} / \mathrm{C}$ respectivement égaux à 0,4 et 0,8 ont été composés. Les résultats des essais triaxiaux permettent d'aboutir aux conclusions suivantes.

Durant la phase déviatoire du comportement, quel que soit le rapport $\mathrm{E} / \mathrm{C} \mathrm{du}$ béton, on observe que le confinement augmente très fortement à la fois la ductilité et la capacité de chargement du béton. La comparaison des trois bétons montre que la diminution du rapport $\mathrm{E} / \mathrm{C}$ augmente le seuil de confinement qui correspond à la transition fragile-ductile du comportement.

Les courbes de comportement volumique du béton montrent que la compaction s'accroit avec l'augmentation de $\mathrm{E} / \mathrm{C}$, en particulier à faible niveau de confinement. On a au premier ordre une corrélation linéaire entre la variation de porosité du béton et sa variation de déformation volumique pour un squelette granulaire donné.

En revanche sous fort confinement, une fois que la matrice cimentaire est fortement endommagée, le comportement volumique et le comportement déviatoire du béton deviennent insensibles au rapport E/C. 
Enfin, la comparaison des états-limites des trois bétons montre comme cela était attendu que pour de faibles niveaux de contrainte moyenne, l'état limite du béton dépend très fortement du rapport $\mathrm{E} / \mathrm{C}$. En revanche, cette dépendance de l'étatlimite du béton vis-à-vis du rapport $\mathrm{E} / \mathrm{C}$ diminue rapidement à mesure que la contrainte moyenne augmente. Au-delà d'une contrainte moyenne critique la courbe d'état-limite devient indépendante du rapport E/C. Cette contrainte moyenne critique est d'autant plus grande que la résistance de la matrice cimentaire est importante.

En résumé, les résultats d'essais présentés dans cet article montrent que sous fort confinement, le béton a un comportement de type empilement granulaire noncohésif sur lequel la résistance de la matrice cimentaire du béton vierge n'a plus d'influence. D'un point de vue applicatif, ces résultats mettent en évidence le peu d'intérêt qu'il y a à augmenter le dosage en ciment des bétons en vue d'augmenter leur capacité de résistance à des sollicitations extrêmes.

\section{Remerciements}

La presse GIGA a été installée au laboratoire 3S-R dans le cadre d'un accord de coopération avec le Centre d'Etudes de Gramat de la Délégation Générale pour l'Armement (CEG, DGA, Ministère Français de la Défense). Cette recherche a été développée avec le support financier du Centre d'Etudes de Gramat (CEG, DGA).

\section{Bibliographie}

ACI 363R-92, State-of-the-art report on high-strength concrete, ACI Manual of Concrete Pratice, Part 1 : Materials and General Properties of Concrete, 55pp., (Detroit, Michigan, 1994), cité dans «Propriétés des bétons », Neville A. M., Eyrolles 2002, 399-400.

ACI 318-89, Building code requirements for structural concrete, ACI Manual of Concrete Pratice, Part 3: Use of Concrete in Buildings - Design, Specifications and Related Topics, 345 pp. (Detroit, Michigan, 1996), cité dans «Propriétés des bétons », Neville A. M., Eyrolles 2002, 399-400.

Akers S. A. and Phillips B. R., «Concrete modelled as un inhomogeneous material: numerical simulations of contact detonation charges ", In $18^{\text {th }}$ International Symposium on the Military Aspects of Blast and Shock, Oberjettenberg, Germany, September 2004

Alexander K. M. and Ivanusec I., «Long term effects of cement $\mathrm{SO}_{3}$ content on the properties of normal and high-strength concrete, Part I. The effect on strength », Cement and Concrete Research, 12, No. 1, pp. 51-60 (1982), cité dans «Propriétés des bétons », Neville A. M., Eyrolles 2002, 255-259.

Bailly P., Tombini C., Le Vu O., «Modélisation de géomatériaux sous sollicitations dynamiques élevées. I : Un tir de pénétration sur cible en béton », Colloque du réseau GEO, Aussois, 2-6 décembre, France, 1996. 
Bazant Z.P., Bishop F.C. and Chang T.P., « Confined compression tests of cement paste and concrete up to $300 \mathrm{Ksi}$ », ACI Journal, vol. 33, p. 553-560, 1986.

Bischoff P. H., Perry S. H., «Compressive behaviour of concrete at high strain rates », Materials and Structures, vol. 24, p. 425-450, 1991.

Bolomey J., Granulation et prévision de la résistance probable des bétons, Travaux 19 (1935) (30), pp. 228-232, 1935.

Burlion N., Compaction des bétons: éléments de modélisation et caractérisation expérimentale, Thèse de doctorat, ENS Cachan, France, 1997.

Burlion N., Pijaudier-Cabot G. and Dahan N., « Experimental analysis of compaction of concrete and motar », Int. J. for Numerical and Analytical Methods in Geomechanics, 25: 1467-1486, 2001.

Buzaud E., Performances mécaniques et balistiques du microbéton MB50, dans le rapport scientifique final «Comportement des ouvrages en dynamique rapide, comportement dynamique du béton » du groupe de recherche formé dans le cadre du réseau de laboratoires «GEO», responsable du projet P. Bailly, 1998.

Chanvillard G., Le matériau béton : connaissances générales, Aléas 1999, 115-139, 1999.

Féret R., Sur la compacité des mortiers hydrauliques, Annales des Ponts et Chaussées, Série 7, vol. $4: 5-164,1892$

Gabet T, Malécot Y., Daudeville L., « Ultimate strength of plain concrete under extreme combined stresses: triaxial and proportional stress paths ", Révue Européenne de Génie Civil, 10(3) : 375-390, 2006.

Gabet T, Malécot Y., Daudeville L., « Triaxial behaviour of concrete under high stresses : Influence of the loading path on compaction and limit states », Cement and concrete research (in press) 2007.

Gilkey H. J., « Water/cement ratio versus strength - another look », J. Amer. Concrete Inst., Part 2, 58, pp 1951-78 (Dec. 1961), cité dans «Propriétés des bétons », Neville A. M., Eyrolles 2002, 255-259.

Hild F., Denoual C., Forquin P., Brajer X., « On the probabilistic-deterministic transition involved in a fragmentation process of brittle materials strains ", Computers \& Structures, vol. 81(12), p. 1241-1254, 2003.

Hopkinson B., « A method of measuring the pressure in the deformation of high explosives or by the impact of bullets ", Philosophic Transactions of the Royal Society, vol. A213, p. $437-452,1914$.

Imran I. , Pantazopoulou S.J., « Experimental study of plain concrete under triaxial stress », ACI Mater. J., 93(6): 589-601, 1996.

Jiang L.H., Huang D.H. and Xie N.X., « Behavior of concrete under triaxial compressivecompressive-tensile stresses », ACI Mater. J., 88(2): 181-185, 1991.

Kakizaki M. , H. Edahiro, T. Tochigi and T. Niki, « Effect of Mixing Method on Mechanical Properties and Pore Structure of Ultra High-Strength Concrete », Katri Report No. 90, pp. 19, 1992, cité dans «Propriétés des bétons », Neville A. M., Eyrolles 2002, 255-259. 
Kupfer H.B., Gerstle K.H., « Behavior of Concrete under Biaxial Stresses », J. of the Eng. Mechanics, Div. 99(4): 853-866, 1973

Mazars J., Application de la mécanique de l'endommagement au comportement non-linéaire et à la rupture du béton de structure, Thèse d'état de l'Université Paris VI, France, 1984

Li H., Pugh D., « Mechanical behavior of materials under pressure », Elsevier, Amsterdam, 1970.

Neville A. M., "Tests on the strength of high-alumina cement concrete ", J. New Zealand Inst. E., 14, No. 3, pp. 73-7, 1959, cité dans «Propriétés des bétons », Neville A. M., Eyrolles 2002, 255-259.

Ollivier J. P., BUIL M., Conception des bétons : la structure poreuse, La Durabilité des bétons, Presses de l'ENPC, Eds., Paris, 1992.

Roy D. M. and Gouda G. R., « Porosity- strength relation in cementitious materials with very high strengths », J. Amer. Ceramic Soc., 53, No. 10, pp. 549-50 (1973), cité dans «Propriétés des bétons », Neville A. M., Eyrolles 2002, 263-269.

Sersale R., Cioffi R., Frigione G. and F. Zenone, Relationship between gypsum content, porosity, and strength of cement, Cement and Concrete Research, 21, No. 1, pp. 120-6 (1991), cité dans «Propriétés des bétons », Neville A. M., Eyrolles 2002, 263-269.

Schmidt J. M., High pressure and high strain rate behaviour of cementitious materials : experiments and elastic/viscoplastic modeling, $\mathrm{PhD}$ thesis, University of Florida, USA, 2003.

Sfer D., Carol I., Gettu R. and Etse G., « Study of the Behavior of Concrete under Triaxial Compression », Journal of Engineering Mechanics, 128 (2): 156-163, february 2002.

Taliercio A., Berra M., Pandolfi A., « Effect of high-intensity sustained triaxial stresses on the mechanical properties of plain concrete », Mag. Concrete Res., 51(6): 437-447, 1999.

Toutlemonde F., Résistance au choc des structures en béton : du comportement du matériau au calcul des ouvrages, Thèse de doctorat de l'ENPC, 1995.

Thiot Ingénierie, la Croix Blanche, 46130 Saint Michel Loubejou, France (Thiot 2004).

Vu X. H., Gabet T., Malecot Y., and Daudeville L., « Experimental analysis of concrete behavior under severe triaxial loading ", in the 2005 Joint ASCE/ASME/SES Conference on Mechanics and Materials, McMat 2005 Mechanics and Materials Conference, Baton Rouge, Louisiana, June 1-3, 2005.

Vu X. H. Caractérisation expérimentale du béton sous fort confinement : influences du degré de saturation et du rapport eau/ciment, Thèse de doctorat, Université Grenoble I, 2007.

Yip W. K., Tam C. T., « Concrete strength evaluation through the use of small diameter cores.», Magazine of Concrete Research, vol. 40, n 143, p. 99-105, 1995.

Yurtdas I., Peng H., Burlion N., Skoczylas F., "Influence of water by cement ratio on mechanical properties of mortars submitted of drying », Cement and Concrete Research, 2005.

Wang C.-Z., Guo Z.-H. and Zhang X.-Q., « Experimental investigation of biaxial and triaxial compressive concrete strength », ACI Mater. J., 84, 92-100, 1987. 
Béton sous fort confinement : effet du rapport eau/ciment 3

Warren T., Fossum A., and Frew D., « Experimental investigation of size effect in concrete fracture under multiaxial compression into low-strength $(23 \mathrm{MPa})$ concrete: target characterization and simulations », Int. J. of Impact Engineering, 30: 477-503, 2004.

William E. M., Akers S. E. and Reed P. A., Constitutive models for the triaxial behaviour of concrete, Report n ${ }^{\circ}$ ERDC/GSL TR-05-16, Geotechnical and Structures Laboratory, U.S. Army, 2005

Zhao H., Gary G., « On the use of SHPB techniques to determine the dynamic behavior of materials in the range of small strains ", Int. J. Solids Structures, vol. 33(23), p. 3363$3375,1996$.

Zukas J.A., « Penetration and perforation of solids », Impact Dynamics, Krieger Publishing Company, 1992. 